\title{
Surgical education in the United States 2010: developing intellectual, technical and human values
}

\author{
Carlos A. Pellegrini
}

Published online: 28 September 2011

(C) Springer-Verlag 2011

\section{Introduction}

Traditional surgical education consisted of a residency lasting 5 years during which the effort was placed on two elements: obtaining information about diseases, and developing technical abilities by observation from the professor and by operating on patients.

Over the last decade there has been a significant change in this paradigm. The need for change comes from several sources. First, the society started to concentrate in the quality of care, including safety. This requires the creation of "systems" and the need to train residents to work on those systems. It also requires that some technical training be obtained before working directly with patients. Second, patients started to demand that their own values be at the center of decision making-which requires special skills on the part of surgeons to understand and to be able to communicate. Third, we saw a revolution in technology and the pace at which the currently developed knowledge has reached a historic height. Therefore, acquiring information is now secondary to learning how to look for new information. Lastly, there has been substantial change in learning technologies with the realization of the principles of adult learning.

In other words, we have moved (educationally speaking) from a system where the professor is viewed at the "center of the learning universe", using authority that emanates from his/her knowledge, to a system in which the learner is placed at the center with the professor acting as a "coach" that provides the necessary tools for the learner to seek the truth. We have moved to a system in which practice and

C. A. Pellegrini $(\bowtie)$

Department of Surgery, University of Washington,

Box 356410, Seattle, WA 981950-6410, USA

e-mail: pellegri@uw.edu learning are done prior to working with patients and where dexterity, and technical abilities are determined objectively and thresholds of performance have been established. Indeed, modern training in surgery today could be defined as the "production of a competent professional who displays cognitive, technical and personal skills required to meet the needs of society".

\section{Evolution of residency training in the last decade}

In the United States three institutions have had, through three historic landmarks, an important role in the evolution of surgical training. The Institute of Medicine, which defined "quality of care"; the Council for Graduate Medical Education which defined the competencies necessary to provide that quality of care and the American Board of Medical Specialties as it redefined the process of certification. Let us briefly review these three landmark decisions and how they are inter-connected.

\section{Quality of care}

The Institute of Medicine defined "quality of care" as the provision of safe, effective, timely, efficient, patientcentered care to all those in need. (Crossing the Quality Chasm, Institute of Medicine, USA).

This definition contains six components to wit: safety, that is to provide care devoid of injury, "primum nonnocere"; efficacy, that is to use methods that yield the best results; efficiency, that is to provide the care with the least utilization of resources; timely that is without the waste of time; patient-centered that is taking into consideration the values of the patients and equitable that is without distinction of financial, political, ethnic or other considerations. 
The competencies

As the concepts above were developed, the Council for Graduate Medical Education and the American Board of Medical Specialties defined the six "competencies" that a medical student, a graduating resident and a physician must have in order to provide quality care. They include: medical knowledge; patient care, which emphasizes respect and compassion for patients; interpersonal skills and communications; professionalism; systems based practice-or the ability to work in teams; and practice based learning or the commitment to seek continuous learning.

In 2003, the Council for Graduate medical education decided to limit the number of hours that a resident could work to 80 per week, with a minimum of $10 \mathrm{~h}$ between duty periods and a maximum stay in the hospital of $30 \mathrm{~h}$. In 2010, the ACGME has restricted further the number of hours, but what is most important has made substantial additional requirements in terms of resident supervision by individuals who have achieved the necessary competency.

\section{Maintenance of certification}

The American Board of Medical Specialties creates the concept of "Maintenance of Certification". This concept emphasizes the need for constant updates to the Board to keep certification current. These updates come from hospitals and reflect the type and quality of practice of an individual who is board certified. There are requirements for the evidence of engagement in continuing learning with self-examinations, and there are requirements for engagement in practice analysis, reporting of outcomes and periodic re-examinations. This new concept does away with the old idea that once training has been completed, the person must take an examination and if successful, be certified for the remainder of his/her professional life.

At its most recent retreat the American Board of Surgery decided to concentrate its efforts in the next few years in three areas: First is to establish yearly milestones for resident progression. This is an acceptance of the concept of "competency" replacing the concept of "time". One must achieve a desired level of training before moving to the next.

Second it will promote the objective measurement of operative skill, a recognition that today we can "measure" the competency with which something is done and define thresholds; and third, it will allow flexibility in the training program, recognizing that the training needs today vary from resident to resident with regards to specialization.

Thus, I believe that now we have a definition of quality of care, and a definition of competencies including milestones to be achieved during residency we can train individuals to proficiency, they are then certified under the new rules that require "maintenance", that is constant renewal of knowledge and skills, which leads to quality of care. Identification of gaps in knowledge and skills, with new training takes us in a complete circle that improves care to patients.

\section{Intellectual development}

We now realize that acquiring information, once one of the two most important things a resident needed to do, is no longer at center of the resident's learning needs. While a certain amount of basic information is important, the most important element is to be well versed on how to acquire old and new information. Secondly, identification of "gaps" in knowledge and skills becomes an important part of the training as it is the development of ways to fill those gaps.

A second important concept in a world in which innovation results in the opportunity to do things differently and to use new devices every day is the ability to maintain a balance between innovation and efficiency. Simply put, the more we do in the exact same way the more efficient (and safe) we become. When we take on something new we lose some of the efficiency, which we hope will be eventually regained. An example would be that of cholecystectomy. Surgeons developed safe and efficient ways of doing it via the open approach. When laparoscopy became available, it was tempting to use it but we had to sacrifice efficiencyeventually to gain a new plateau.

There is a new science that we must instill in our residents, which is that of "adaptive expertise" which essentially deals with the ability to be able to strike a balance in our work, that while allowing for the development of expertise does not stifle innovation, while at the same time not only introduces enough innovation to keep growing the science and techniques of surgery but also allows for the development of efficiency. In other words we do not want our surgeons to become either "frustrated experts" (someone trying something new every day) or remain "routine experts" someone who never changes.

Innovation does not only present a challenge to efficiency. But it also offers a substantial challenge to education. Indeed, in the past, innovation was a "sporadic" phenomenon occurring at a point in time every so often. One can only think of great developments such as antisepsis, anesthesia, blood transfusion, etc., and realize that between major changes, there was a substantial period of time that allowed training programs to adapt to the new ways. With increasing pace in innovation, the ability to develop learning tools and educational processes is much more difficult as they become outdated almost simultaneously with their development. It is what has been called the "white water rafting" of education. 


\section{Skill acquisition}

As previously mentioned, in the past surgeons developed skills by observation and by working directly with patients. However, there is a price to pay because of the existence of a learning curve. Several studies have shown that during the first 20-30 cases the patient experiences a greater risk and the time to complete the operation is longer. In addition, there are simply not enough cases for the residents to learn all techniques necessary to achieve the minima required to graduate. One of the greatest advances of the XXI century has been the development of simulation and simulation centers.

From an educational perspective, simulation offers substantial advantages: the method is learner centered (as opposed to patient-centered), and therefore it is possible to repeat as needed and to do it without negative consequences. In addition, it is usually possible to measure performance in an objective way and to provide immediate feedback to the learner. Lastly, uncommon problems and errors can be actually learned in simulated environments without exposing a patient to unnecessary risks.

Simulation techniques are now used routinely to teach team training, to enhance the ability to work in inter-professional groups, to practice communication skills, and to improve the ability of surgeons to work with the rest of the health care team as a leader.

We can assess progress in the learning of skills by sophisticated techniques that can read the work of one hand independent of the other by measuring the movement of the tips of the tools utilized or by measuring the movements of the retina. These data allow a trainer to determine the level of expertise that the trainee has achieved at a given point in time facilitating training and reaching defined thresholds of skill.

\section{Developing the total person}

One of the fundamental changes that we have seen in the training of residents is the importance placed today in the development of the "total person". Work hour limitations, protection of personal time, and a commitment to some form of balance between pure service to patients, education, and emotional development have become integral parts of the residency training. To that end, residents learn fatigue recognition and management, they are trained in aspects of emotional intelligence, leadership, and communication styles, they practice "giving bad news to patients" and disclosure of errors in simulated environments or with the trained actors and other similar things.

In this context, educators have learned to appreciate the value of role modeling in the development of the resident. This has been defined more recently as the "hidden curriculum" to define a curriculum that, while not "formal" or "written", has the potential to shape the character of the graduates of the program. Perhaps the figure of the professor, once cherished primarily as "the repository of all knowledge", is now slowly becoming the figure of an individual the residents consciously or unconsciously try to emulate. This evolution from the professor being viewed as the fountain of knowledge to the professor being viewed as someone, one wishes to emulate was best described by the Italian jurist Salvatore Satta, [Soliloqui e colloqui di un giurista. 1969], when describing the ideal professor "Essere professore significa una sola cosa: insegnare con lo scritto, con la parola e con la vita. Ed in questi tre indissociabili aspetti sta la sua sacerdotale grandezza”.

Conflict of interest None. 